Review Article

\title{
Monoclonal Antibody Therapies in Multiple Myeloma: A Challenge to Develop Novel Targets
}

\author{
Hiroko Nishida $\mathbb{D}^{1}$ and Taketo Yamada ${ }^{1,2}$ \\ ${ }^{1}$ Department of Pathology, Keio University, School of Medicine, Tokyo 160-8582, Japan \\ ${ }^{2}$ Department of Pathology, Saitama Medical University, Faculty of Medicine, Saitama 350-0495, Japan \\ Correspondence should be addressed to Hiroko Nishida; hiroko@a2.keio.jp
}

Received 10 May 2019; Accepted 13 September 2019; Published 3 November 2019

Guest Editor: Xuelei Ma

Copyright (c) 2019 Hiroko Nishida and Taketo Yamada. This is an open access article distributed under the Creative Commons Attribution License, which permits unrestricted use, distribution, and reproduction in any medium, provided the original work is properly cited.

\begin{abstract}
The treatment options in multiple myeloma (MM) has changed dramatically over the past decade with the development of novel agents such as proteasome inhibitors (PIs); bortezomib and immunomodulatory drugs (IMiDs); thalidomide, and lenalidomide which revealed high efficacy and improvement of overall survival (OS) in MM patients. However, despite these progresses, most patients relapse and become eventually refractory to these therapies. Thus, the development of novel, targeted immunotherapies has been pursued aggressively. Recently, next-generation PIs; carfilzomib and ixazomib, IMiD; pomalidomide, histone deacetylase inhibitor (HDADi); panobinostat and monoclonal antibodies (MoAbs); and elotuzumab and daratumumab have emerged, and especially, combination of mAbs plus novel agents has led to dramatic improvements in the outcome of MM patients. The field of immune therapies has been accelerating in the treatment of hematological malignancies and has also taken center stage in MM. This review focuses on an overview of current status of novel MoAb therapy including bispecific T-cell engager (BiTE) antibody (BsAb), antibody-drug conjugate (ADC), and chimeric antigen receptor (CAR) T cells, in relapsed or refractory MM (RRMM). Lastly, investigational novel MoAb-based therapy to overcome immunotherapy resistance in MM is shown.
\end{abstract}

\section{Introduction}

The treatment options in MM has changed dramatically over the past decade with the emergence of novel agents including proteasome inhibitors (PIs, bortezomib) and immunomodulatory drugs (IMiDs, thalidomide and lenalidomide) and exerts a remarkable impact on the outcome of MM patients [1-3]. However, most patients who achieve a prolonged response following initial therapy may ultimately relapse or become refractory. Thus, the development of novel, targeted immunotherapies has been pursued aggressively. Recently, next-generation PIs (carfilzomib and ixazomib) [4-9], IMiDs (pomalidomide) [10-12], histone deacetylase inhibitor (HDACi, panobinostat) [13-15], and the monoclonal antibodies (MoAbs, elotuzumab and daratumumab) have emerged and further improved the clinical outcome in MM patients who are refractory to prior treatments [12, 16-36]. Importantly, MM remains a chronic disease, so in order to overcome the disease relapse, ongoing challenges to pursue novel therapeutic strategies as well as predictive biomarkers for response or resistance to immunotherapies are required. Furthermore, these novel therapies are expected to be potentially useful in the treatment options for patients who are ineligible for autologous stem cell transplantation (SCT) followed by high-dose chemotherapy [37].

Monoclonal antibody (MoAb) therapies have been accelerating and shown to be able to improve the outcome of cancers [38]. In hematological malignancies, rituximab, a chimeric murine/human anti-CD20 monoclonal $\operatorname{IgG}_{1 \kappa}$ antibody or of atumumab, a humanized anti-CD20 monoclonal $\mathrm{IgG}_{1 \kappa}$ antibody, targeting CD20 on B cells, is currently indicated for the treatment of B-cell non-Hodgkin's lymphoma (NHL) and chronic lymphocytic leukemia (CLL). It exerts significant activity in combination with cytotoxic anticancer drugs $[38,39]$. 
Although these progresses in immune therapies and their application for the treatment of MM have not succeeded until recently, these therapeutic strategies have finally attained a breakthrough with the development of the MoAb therapies targeting surface molecules, expressed in MM cells, such as elotuzumab, a humanized anti-CS1/SLAMF7 monoclonal antibody, and daratumumab, a humanized antiCD38 monoclonal antibody, both of which have been approved in the treatment of relapsed or refractory MM (RRMM) patients who received at least three prior therapies including PIs and iMiDs [40-43]. Herein, we review an overview of the current status of MoAb therapies in RRMM. In addition, we introduce investigational novel MoAb therapies in RRMM and show future direction toward immunotherapy resistance in MM.

\section{Monoclonal Antibodies (MoAbs) in MM}

Potential MoAbs target various kinds of antigens including growth factors, signaling molecules, cell surface proteins, and molecule of adhesion. Ideally, these MoAb-therapeutic targets should be predominantly expressed on a majority of MM cells, but not on normal hematopoietic cells or nonhematopoietic tissues. MoAb therapies involve several mechanisms including direct cytotoxic effects, antibodydependent cellular cytotoxicity (ADCC), complement-dependent cellular cytotoxicity (CDC), and interference with cell-to-cell interactions [40-43]. Other mechanisms include the use of intracellular toxins or radioactive isotopes conjugated to MoAbs after its internalization into tumor cells, which reveal cytotoxicity against tumor cells beyond those bearing MoAb target antigens [40-43].

2.1. CD20 and Rituximab. CD20 is a transmembrane phosphoprotein expressed on committed B lymphoid cells through the all stages of their development, but its expression is reduced in plasma cells. Rituximab, a chimeric murine/human anti-CD20 monoclonal $\operatorname{IgG}_{1 \kappa}$ antibody targeting CD20 on B cells, is currently indicated for the treatment of B-cell non-Hodgkin's lymphoma (NHL) and chronic lymphocytic leukemia (CLL) [39]. It exerts significant activity in combination with cytotoxic anticancer drugs. However, CD20 is present only in a few plasma cells and is absent in most of plasma cells in MM. Therefore, few selected MM patients achieved only minimal responses (MD) [44-46]. Moreover, MM cells express increased levels of complement-inhibitory proteins which result in the reduction of CDC via rituximab against tumor cells.

2.2. CS1/SLAMF7 and Elotuzumab. Elotuzumab is a humanized IgG $_{1}$ monoclonal antibody which targets SLAMF7, known as CS1, a glycoprotein, intensely expressed on MM cells and normal plasma cells as well as natural killer (NK) cells. It induces cytotoxicity against MM cells via NK cell-associated ADCC, NK cell activation, and inhibition of the interaction between MM cells and bone marrow stromal cells (BMSCs). Elotuzumab revealed intensive anti-MM efficacy and safety profiles when combined with IMiDs or
PIs in previously treated RRMM [12, 16-21] (Table 1). The phase II results demonstrated that elotuzumab in combination with lenalidomide plus dexamethasone $(\mathrm{Rd})$ in patients with RRMM showed safety and efficacy which was better than previously noted with Rd [17, 18]. Moreover, results of the phase III trial ELOQUENT-2 clearly proved the benefit of adding elotuzumab to $\mathrm{Rd}$ for the treatment of RRMM [18]. The overall response rates (ORRs) were $79 \%$ for the elotuzumab group and $66 \%$ for the control group; the PFS rate was 68 vs. $57 \%$ for the elotuzumab and control groups at 1 year and 41 vs. $27 \%$ at 2 years; the median PFS was 19.4 vs. 14.9 months for the elotuzumab and control groups [19]. Based on the results of these trials, elotuzumab attained food and drug administration (FDA) approval in 2015 in combination with $\mathrm{Rd}$ for the treatment of RRMM patients, who previously received two or three prior therapies. A phase III randomized study of Rd with or without elotuzumab in previously treated MM patients is currently ongoing. Phase II trials of elotuzumab plus pomalidomide and dexamethasone (EPd) vs Pd in 117 patients who received $>2$ prior therapies revealed that after a follow-up period of 9 months, EPd had a longer median PFS (10.3 vs 4.7 month) and a better ORR (53 vs 26\%) [12]. Phase II trials of elotuzumab plus bortezomib and dexamethasone (EBd) vs $\mathrm{Bd}$ in 77 patients who had received one to three prior therapies showed that EBd had a longer median PFS (9.7 vs 6.9 months). However, there was no deference in ORR between EBd group and Bd group (66\% vs 63\%) [20, 21].

2.3. CD38 and Daratumumab. Daratumumab is a humanized $\mathrm{IgG}_{1}$-kappa monoclonal antibody targeting CD38, which is $46-\mathrm{kDa}$ type II transmembrane glycoprotein, broadly expressed on plasma cells as well as lymphoid cells, myeloid cells, and nonhematopoietic tissues. It is also expressed in OCs. CD38 retains multiple functions including ectoenzymatic activity, signal transduction, and receptormediated regulation of cell adhesion $[22,23]$. In preclinical studies, daratumumab revealed anti-MM cytotoxicity through multiple mechanisms including ADCC, ADCP, $\mathrm{CDC}$, and direct apoptosis via FcR-mediated cross linking of daratumumab in vitro [24-26] (Table 2). Of note, no difference was revealed in daratumumab-associated ADCC or CDC between newly diagnosed and RRMM patients. The level of CD38 expression in MM cells was reported to be related to daratumumab-associated ADCC and CDC [2426]. Moreover, daratumumab has several effects on the immune system. It increases CD8+/CD4+ and CD8+ Treg ratios as well as memory $\mathrm{T}$ cells, while decreasing naïve T cells, which enhance the overall immune response to MM cells [27].

Daratumumab revealed anti-MM efficacy as monotherapy as well as in combination with novel agents in heavily pretreated RRMM patients, which resulted in FDA approval in 2015. The GEN501 and SIRIUS trials demonstrated that daratumumab is active as monotherapy in RRMM patients [28, 29]. It showed improved ORRs regardless of refractoriness to prior therapies including PIs and IMiDs (31\%). [30]. Phase III Castor trials revealed that 
TABLE 1: Summary of clinical trials in anti-CS1/SLAMF7 antibody in relapsed/refractory MM.

\begin{tabular}{|c|c|c|c|c|c|c|}
\hline References & & Phase & Regimen & ORR (\%) & PFS (mo) & OS \\
\hline Richardson et al. [17] & & 2 & Elo + Rd & $84.00 \%$ & NA & NA \\
\hline Lonial et al. [18] & ELOAUENT2 & 3 & Rd \pm Elo & $79 \%$ vs $66 \%$ & $19.4 \mathrm{mo}$ vs $14.9 \mathrm{mo}$ & NA \\
\hline Dimopoulos et al. [12] & & 2 & $\mathrm{Pd} \pm$ Elo & $53 \%$ vs $26 \%$ & $10.3 \mathrm{mo}$ vs $4.7 \mathrm{mo}$ & NA \\
\hline Jakubowiak et al. [20] & Elo-Bd & 2 & Bd \pm Elo & $66 \%$ vs $63 \%$ & $9.7 \mathrm{mo}$ vs $6.9 \mathrm{mo}$ & 1 yr $85 \%$ vs $74 \%$ \\
\hline Zonder et al. [16] & Phase1 Elo & 1 & Elo Dose Escalation & MTD not identified & NA & NA \\
\hline Jakubowiak, et al. [21] & Elo-Bd & 1 & Elo + Bd & $48.00 \%$ & $9.5 \mathrm{mo}$ & NA \\
\hline Lonial, et al. [19] & Elo-Rd & 1 & Elo + Rd & $82.00 \%$ & NA & NA \\
\hline
\end{tabular}

MM, multiple myeloma; Elo, elotuzumab; Rd, lenalidomide plus dexamethasone; Pd, pomalidomide plus dexamethasone; Bd, bortezomib plus dexamethasone, NA, not available; MTD, maximum tolerated dose.

TABLE 2: Summary of clinical trials in anti-CD38 antibody in relapse/refractory MM.

\begin{tabular}{|c|c|c|c|c|c|c|}
\hline References & & Phase & Regimen & ORR (\%) & PFS (mo) & OS \\
\hline Lokhorst et al. [28] & GEN501 & $1 / 2$ & Dara monotherapy & $36 \%$ & $5.6 \mathrm{mo}$ & 1 yr $77 \%$ \\
\hline Lonial et al. [29] & SIRIUS & 2 & Dara monotherapy & $17 \%$ & $3.7 \mathrm{mo}$ & 1 yr $65 \%$ \\
\hline Spencer et al. [32] & CASTOR & 3 & $\mathrm{Bd} \pm$ Dara & $83 \%$ vs $63 \%$ & $1.5 \mathrm{yr} 48 \%$ vs $8 \%$ & NA \\
\hline Palumbo et al. [31] & CASTOR & 3 & $\mathrm{Bd} \pm$ Dara & $83 \%$ vs $63 \%$ & $1 \mathrm{yr} 61 \%$ vs $27 \%$ & NA \\
\hline Dimopoulos et al. [33] & POLLUX & 3 & $\mathrm{Rd} \pm$ Dara & $93 \%$ vs $76 \%$ & 1 yr $83 \%$ vs $60 \%$ & NA \\
\hline Dimopoulos et al. [34] & POLLUX & & $\mathrm{Rd} \pm$ Dara & $93 \%$ vs76\% & 2 yr $68 \%$ vs $41 \%$ & NA \\
\hline Chari et al. [35] & EQULLEUS & $1 \mathrm{~b}$ & $\mathrm{Pd} \pm$ Dara & $60 \%$ & $1 \mathrm{yr} 42 \%$ & 1 yr $89 \%$ \\
\hline
\end{tabular}

MM, multiple myeloma; Dara; daratumumab, Rd, lenalidomide plus dexamethasone; Bd, bortezomib plus dexamethasone; Pd, pomalidomide plus dexamethasone; NA, not available; MTD, maximum tolerated dose.

daratumumab significantly improved ORR, PFS, and time to progression (TTP) in combination with Bd, ORR ( $83 \%$ vs $63 \%$ ), the 12 -month rate of PFS (61\% vs $27 \%$ ), and TTP at 12 months (65\% vs 29\%) [31]. Another phase III Castor study also revealed a significant benefit of D-Bd over Bd regardless of treatment history or cytogenetic risk [32]. Phase III POLLUX trials demonstrated remarkable efficacy of daratumumab in combination with lenalidomide plus dexamethasone (DRd) in patients with RRMM $[33,34]$. The ORR was $92.9 \%$ in DRd group versus $72.9 \%$ in Rd group. DRd improved PFS compared with Rd with 12-month PFS rates of $83.2 \%$ in DRd group versus $60.1 \%$ in Rd group and 24 -month PFS rate of $68.0 \%$ versus $40.9 \%$, restrictively $[33,34]$. The EQUULEUS study led to the FDA approval of daratumumab in combination with Pd in 2017 for RRMM patients who have received 2 or more prior line of therapy including lenalidomide and a PI. The median PFS was 8.8 months, the 12 -month PFS rate was $42 \%$, the median OS was 17.5 months, and the median 12 -month survival rate was $66 \%[35]$.

\section{Novel Target Antigens in MoAb Therapies in MM}

3.1. CD38 and Isatuximab. Isatuximab is a chimeric $\operatorname{IgG}_{1^{-}}$ kappa anti-CD38 monoclonal antibody which selectively binds to a unique epitope on human $\mathrm{CD} 38$ receptor and elicits anti-MM activity by direct apoptosis, ADCC, and ADCP [47]. CDC was triggered in less than half of MM patients with high levels of CD38 in MM cells. A phase $1 \mathrm{~b}$ open-label, dose escalation study showed that 57 patients who had received at least one prior line of therapy attained ORR of $52 \%$ by isatuximab plus Rd in 42 evaluable lenalidomide-refractory patients, and overall median PFS was
8.5 months [48]. Another phase $1 \mathrm{~b}$ study of isatuximab plus $\mathrm{Pd}$ in patients with RRMM who had received more than 2 prior therapies also revealed that ORR was $62 \%$; median duration of response was 18.7 months; and PFS was 17.6 months [49].

3.2. Interleukin-6 (IL6) and Siltuximab. Interleukin-6 is an important cytokine for the growth and survival of MM cells. It is chiefly produced by BMSCs and increased by several cytokines. A chimeric anti-IL-6 antibody, siltuximab, revealed cytotoxicity in MM patients who was refractory to dexamethasone [50]. In addition, it increased cytotoxicity with $\mathrm{Bd}$ in combination, whereas in a phase 2 randomized study of siltuximab plus bortezomib, the addition of siltuximab to bortezomib did not appear to improve PFS or OS in refractory MM patients [51]. The other study showed that there were no responses to siltuximab but combination therapy with dexamethasone yielded a partial or minimal response rate of $23 \%$, in dexamethasone-refractory MM [51].

3.3. PD-1/PD-L1 Inhibitors. Programmed cell death protein 1 (PD-1)/programmed cell death ligand 1 (PD-L1) pathway is a negative regulator of immune activation [52]. Recently, there are discrepancies concerning programmed death $\mathrm{PD}$ L1 expression on plasma cells in MM. Several data demonstrated that PD-L1 is overexpressed on MM plasma cells but not on normal plasma cells [53-56]. It was reported that PD-L1 expression on plasma cells was associated with increased risk of progression from smoldering MM (SMM) into MM [57], whereas other reports showed that no difference was detected in PD-L1 expression on plasma cells between MM, SMM, monoclonal gammopathy of undetermined significance (MGUS), and healthy individuals 
[58, 59]. Similarly, discordant results were reported regarding PD-1 expression on immune cells, including T cells and NK cells in MM. Paiva et al. showed that PD-1 was overexpressed on CD4+ and CD8+ T cells in MM patients [58]. Benson et al. demonstrated that PD-1 expression was increased on NK cells from MM patients, compared with normal NK cells, whereas Paiva et al. demonstrated there was no difference between these cells $[58,60]$.

Among hematological malignancies, antibody blockade of the PD-1/PD-L1 pathway is a highly effective therapeutic approach for patients with classical Hodgkin lymphoma, $97 \%$ of which typically exhibits an overexpression of PD-L1 due to the alteration in chromosome 9p24.1 (54). Therefore, the PD-1/PD-L1 axis is a good target for MoAbs, leading immune cells to kill tumor cells. The use of nivolumab, a human IgG4 MoAb which blocks the interaction with PD-L1 and PD-L2 by binding to the PD-1 receptor on activated immune cells, was approved by FDA in 2016 for the treatment of relapsed or progressed Hodgkin lymphoma [52]. However, the outcome of checkpoint blockade by monotherapy with PD-1/PD-L1 inhibitors is unsatisfactory in $M M$, compared with solid tumors due to the reduced immune dysfunction in $\mathrm{MM}[58,59]$. In contrast, lenalidomide enhances the effect of PD-1/PD-L1 blockade on both T cell- and NK cell-mediated cytotoxicity. The combination therapy of lenalidomide plus PD-1/PD-L1 inhibitors increased interferon $\gamma$ by BM-derived effector cells in MM and was associated with increased apoptosis of MM cells, suggesting synergistic cytotoxic effects [56, 61, 62]. There are only limited data from clinical trials of PD1/PDL1 MoAbs in MM patients. The phase Ib trial of nivolumab monotherapy in 27 RRMM patients showed the stabilization of disease status in 17 patients, lasting a median of 11.4 weeks [63]. A phase I study of pembrolizumab with Rd in RRMM patients revealed a partial response rate of $50 \%$ [61, 62, 64, 65]. A phase 3 study of the combination of Rd with or without pembrolizumab was performed in transplant ineligible newly diagnosed MM patients (KEYNOTE-185 trial) $[61,62,64]$. A Phase 3 study of the combination of Pd with or without pembrolizumab was conducted in the KEYNOTE183 trial, and it led FDA to discontinue the trial, due to increased risk of death of patients $[61,62,65]$.

3.4. Bispecific T-Cell Engager (BiTE) Antibodies (BsAb). Bispecific T-cell engager (BiTE) antibodies (BsAbs) are constructs, composed of 2 linked MoAbs which target 2 epitopes. One arm of antibody, scFvs, binds to $\mathrm{CD} 3$ on tumor-specific T cells, while the other arm binds to tumorspecific antigen on tumor cells $[66,67]$. Cross linkage of $\mathrm{T}$ cells to the tumor cells causes $\mathrm{T}$ cells to release cytotoxic molecules such as perforin, which creates transmembrane pores in tumor cells, and granzyme B, which initiates apoptosis toward tumor cells. In addition, cytokine production from $\mathrm{T}$ cells activates its proliferation to kill tumor cells. BsAbs are characterized by small size $(5 \mathrm{kDa})$, which induces high efficacy toward tumor cells, but its serum half-life is short $[66,67]$. B-cell maturation antigen (BCMA) belongs to tumor necrosis factor superfamily member 17 , also named
"TNFRSF17 or CD269," which is uniformly expressed in malignant plasma cells but not in normal essential nonhematopoietic tissues, and only restricted expression is detected in normal hematopoietic cells including normal plasma cells and mature B lymphocytes. Thus, it is a highly plasma cell specific antigen and has a central role in regulating B-cell maturation and differentiation into plasma cells by engaging a proliferation-inducing ligand (APRIL) cells. This expression pattern leads to the development of BCMAspecific mAbs, BsAbs, antibody-drug conjugates (ADCs), and chimeric T cell receptor (CAR) T cells [68-70]. BsAb, BI836909 (AMG420), the first bispecific scFv, simultaneously binds to CD3+ T cells and BCMA + MM cells which make a cross linking between both cells to induce cytolytic synapse, activate T cells, and lyse BCMA + MM cells. In phase I study in RRMM patients, it exhibited potent and high efficacy by depleting BCMA + MM cells [68-70]. CD3xCD38 BsAb, engineered to direct $\mathrm{T}$ cells to $\mathrm{CD} 38$ on tumor cells, was also developed. The phase 1 multicenter study of GBR1342 is underway [71].

3.5. Antibody-Drug Conjugates (ADCs). Antibody-drug conjugate is composed of recombinant MoAbs, bound to cytotoxic chemical agents through synthetic chemical linkers. MoAbs bind to the cell surface antigen on tumor cells and are internalized with the chemicals. Thus, the cytotoxic chemicals are released and transported from lysosome into cytosol to kill tumor cells [72]. GSK2857916 is a humanized and $\operatorname{IgG}_{1}$ MoAb with high affinity to BCMA with afucosylated Fc linked to auristatin F noncleavable linker, maleimidocaproyl. In preclinical study, it binds to BCMA + MM cells and induces G2/M arrest and apoptosis by the activation of caspase $3 / 7$ and 8 . The naked form of ADC augmented effector-mediated cytotoxicity including ADCC and ADCP against patient MM cells [72]. In MM xenograft models, GSK2857916 depletes MM cells but surrounding BCMA-BM accessory cells remain unharmed. Its cytotoxicity is further increased by GSK2857916 plus lenalidomide in combination. In phase 1 study of GSK2857916 in RRMM patients, GAK2857916 monotherapy revealed a $60 \%$ response rate and median PFS of 7.9 months [73, 74]. Anti-BCMA approaches, alone or in combination with iMIDs or immune checkpoint inhibitors, will be evaluated in clinical trials in MM [70].

3.6. Chimeric Antigen Receptor (CAR) T Cells. CARs are fusion proteins incorporating an antigen-recognition domain and T-cell signaling domain. $\mathrm{T}$ cells are genetically modified to express CARs, which specifically recognize target antigens on tumor cells [75-77]. CAR T-cell therapy has already approved by FDA and European Medicine Agency (EMA) for the treatment of relapsed of refractory Bacute lymphoblastic leukemia (ALL) and diffuse large B cell lymphoma (DLBCL) [75-77]. CAR-expressing T cells targeting CD19 revealed efficacy in patients with acute lymphoblastic leukemia (ALL) or B-cell NHL. This success of CAR-T cells against leukemia or lymphoma has encouraged the development of CAR-T therapies for MM. In the first 
TABLE 3: Investigational monoclonal antibodies in MM.

\begin{tabular}{lccc}
\hline Target molecule & mAb & Type & Clinical trials \\
\hline CD138 & Indatuximab ravtansine & ADC & Inda \pm Rena ORR 78\% vs 4\% \\
CD56 & Lorvotuzumab & ADC & Lorv+/Rd ORR 56\% vs 7\% \\
CD40 & Dacetuzumab, lucatumumab & Humanized & Luattained prolonged PR \\
CD74 & Milatuzumab & Humanized & Nobective responses \\
BAFF & Tabalumab & Humanized & Bd Taba; ORR 44\% \\
BCMA & GSK2857916 & ADC & MTD not determined \\
GRP78 & PAT-SM6 & Humanized & MTD not determined \\
IGF-1R & AVE1642 & Humanized & No objective responses \\
ICAM-1 & BI-505 & Humanized & No objective responses \\
CD26 & YS110 (huCD26mAb) & Humanized & Best responses 50\% \\
\hline
\end{tabular}

ADC, antibody-drug conjugate; Lena, lenalidomide; Inda, indatuximab ravatansine, Rd, lenalidomide plus dexamethasone; Lorv, lorvotuzumab; Luc, lucatumumab; PR, partial response; Bd, bortezomib + dexamethasone; Taba, tabalumab; MTD, maximum tolerated doses.

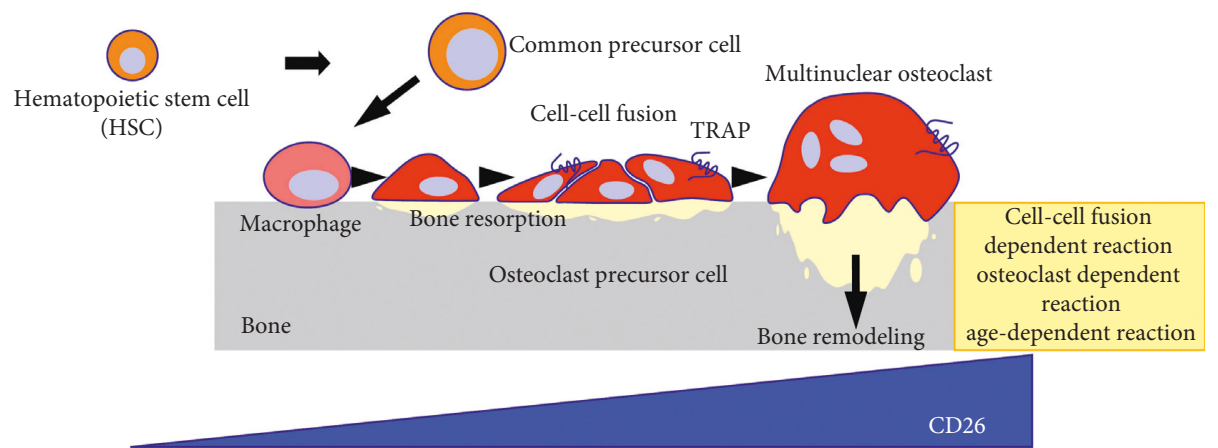

FIgURE 1: CD26 in human osteoclast development CD26 expression is increased during human osteoclast (OC) development.

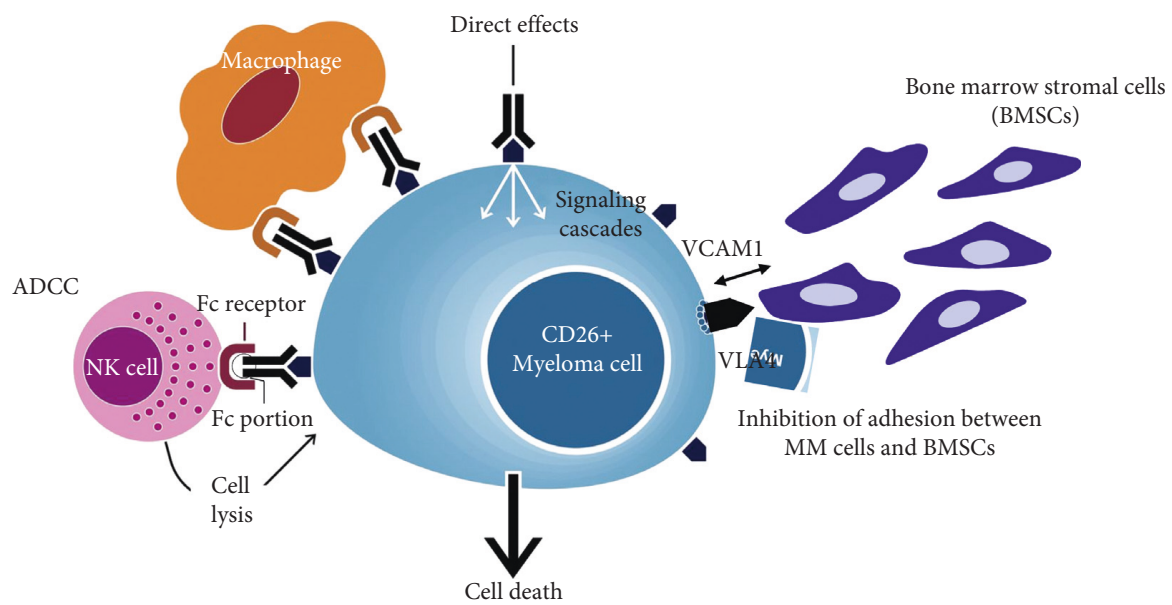

Figure 2: Humanized anti-CD26 monoclonal antibody (huCD26mAb): mechanisms of action huCD26mAb inhibits CD26 + MM cell growth chiefly via ADCC.

human clinical trials, Carpenter et al. designed the first novel CAR targeting BCMA in MM and demonstrated CARBCMA T cells had powerful activity against MM that was resistant to standard therapies [78, 79]. Moreover, bb2121 was produced by transducing autologous $\mathrm{T}$ cells with a lentiviral vector encoding a second-generation CAR incorporating an anti-BCMA single-chain variable fragment, CD137 costimulatory motif, and a CD3-zeta signaling domain [80]. A phase 1 clinical study of bb2121 in heavily pretreated RRMM patients revealed that $85 \%$ of the patients had a clinical response lasting a median of 10.9 months without any ongoing MM therapies [80]. Currently, CAR$\mathrm{T}$ cell therapy for MM remains experimental. CAR-T cell therapy is a potentially life-threatening therapeutic approach, which needs to be administrated in experience hospitals. Now, phase 3 trials are just starting for RRMM in 
2019. In addition, novel CARs targeting alternative plasma cell antigens including CD38, CD44v6, and SLAMF7(CS) are being developed [81, 82].

\section{Experimental Research in Novel MoAb Therapy in RRMM}

4.1. Investigational MoAbs. Target antigens for MoAb are either cell surface membrane proteins or soluble factors including cytokines or chemokines expressed or secreted in MM cells. Their functions include MM cell growth, cellular adhesion, angiogenesis, apoptosis, and cell-to-cell contact between MM cells microenvironmental cells. Investigational mAbs targeting CD138, CD56, CD40, CD74, BAFF, BCMA, GRP78, IGF-1R, and ICAM-1 are preclinically developed, and several of them are in clinical trials [83-92] (Table 3).

\subsection{Humanized Anti-CD26 Monoclonal Antibody} (huCD26mAb). CD26 is a $110 \mathrm{kDa}$ transmembrane glycoprotein with dipeptidyl peptidase (DPPIV) activity, which is widely expressed in various normal cells such as T lymphocytes, natural killer (NK) cells, basophils, eosinophils, endothelial cells, and epithelial cells [93-96]. In addition, CD26 is expressed in several tumor cells including malignant lymphoma, mesothelioma, renal cell carcinoma, and hepatocellular carcinoma and is involved in T-cell activation and tumorigenesis [97, 98]. We have recently characterized CD26 as a potential therapeutic target for the treatment of MM [99]. We identified CD26 expression in human osteoclasts (OCs) in healthy individuals (Figure 1). Its expression is further increased in osteoclasts in osteolytic bone tumors including MM, adenocarcinoma, lung cancer, and osteosarcoma. huCD26mAb, a humanized $\operatorname{IgG}_{1}$ monoclonal antibody that directly targets CD26, inhibits human OC differentiation in vitro and in vivo analysis [99]. In the bone marrow tissue of MM patients, we found that CD26 was present in plasma cells around OCs or endothelial cells. In vitro immunostaining or flow cytometry studies revealed that although CD26 expression was low or absent on MM cell lines cultured alone, it was intensely and uniformly expressed on MM cell lines cocultured with OCs [100]. The augmented CD26 expression in MM cells was exploited to enhance cytotoxicity of huCD26mAb chiefly via a substantial increase in antibody-dependent cytotoxicity (ADCC) against MM cells, direct effects or inhibition of the adhesion between MM cells and BM stromal cells (BMSCs) (Figure 2). Moreover, huCD26mAb in combination with the existing standards of care including bortezomib and lenalidomide synergistically enhanced huCD26mAb-induced ADCC activity against CD26+MM cells compared with each agent alone [100]. Lastly, therapeutic effect of huCD26mAb against MM cell growth and its related osteolytic lesion was also validated in vivo, using a xenograft model: an intrabone tumor model of MM. Our preclinical results demonstrated that huCD26mAb elicited significant anti-MM efficacy by impairing both CD26 + MM cells and
OCs in vivo, suggesting that CD26 could be an ideal therapeutic target of antibody-based therapy in RRMM [100].

\section{Conclusion}

During the last decades, therapeutic strategies in MM have dramatically changed. MoAbs act synergistically with backbone regimens including iMIDs, PIs, or HDACi and have benefits to overcome resistance to prior therapies. The future treatment options of MM to overcome resistance are promising by combination with MoAbs plus these novel agents, check point inhibitors or CAR T-cell therapy.

\section{Conflicts of Interest}

The authors declare they have no conflicts of interest.

\section{Supplementary Materials}

CD26 in human osteoclast development humanized antiCD26 monoclonal antibody (huCD26mAb): mechanisms of action summary of clinical trials in anti-CS1/SLAMF7 antibody in relapsed/refractory MM. Summary of clinical trials in anti-CD38 antibody in relapsed/refractory MM. Investigational monoclonal antibodies in MM. (Supplementary Materials)

\section{References}

[1] A. Palumbo and K. Anderson, "Multiple myeloma," New England Journal of Medicine, vol. 364, no. 11, pp. 1046-1060, 2011.

[2] P. Moreau and E. de Wit, "Recent progress in relapsed multiple myeloma therapy: implications for treatment decisions," British Journal of Haematology, vol. 179, no. 2, pp. 198-218, 2017.

[3] C. Varga, J. P. Laubach, K. C. Anderson, and P. G. Richardson, "Investigational agents in immunotherapy: a new horizon for the treatment of multiple myeloma," British Journal of Haematology, vol. 181, no. 4, pp. 433-446, 2018.

[4] H. Avet-Loiseau, R. Fonseca, D. Siegel et al., "Carfilzomib significantly improves the progression-free survival of highrisk patients in multiple myeloma," Blood, vol. 128, no. 9, pp. 1174-1180, 2016.

[5] A. K. Stewart, S. V. Rajkumar, M. A. Dimopoulos et al., "Carfilzomib, lenalidomide and dexamethasone for relapsed multiple myeloma," New England Journal of Medicine, vol. 372, no. 2, pp. 142-152, 2015.

[6] P. Moreau, M.-V. Mateos, J. R. Berenson et al., "Once weekly versus twice weekly carfilzomib dosing in patients with relapsed and refractory multiple myeloma (A.R.R.O.W.): interim analysis results of a randomised, phase 3 study," The Lancet Oncology, vol. 19, no. 7, pp. 953-964, 2018.

[7] P. Moreau, T. Masszi, N. Grzasko et al., "Oral ixazomib, lenalidomide, and dexamethasone for multiple myeloma," New England Journal of Medicine, vol. 374, no. 17, pp. 1621-1634, 2016.

[8] M. A. Dimopoulos, F. Gay, F. Schjesvold et al., "Oral ixazomib maintenance following autologous stem cell transplantation (TOURMALINE-MM3): a double-blind, randomised, placebo-controlled phase 3 trial," Lancet, vol. 393, no. 10168, pp. 253-264, 2019. 
[9] C. Touzeau and P. Moreau, "Ixazomib in the management of relapsed multiple myeloma," Future Oncology, vol. 14, no. 20, pp. 2013-2020, 2018.

[10] J. S. Miguel, K. Weisel, P. Moreau et al., "Pomalidomide plus low-dose dexamethasone versus high-dose dexamethasone alone for patients with relapsed and refractory multiple myeloma (MM-003): a randomised, open-label, phase 3 trial," The Lancet Oncology, vol. 14, no. 11, pp. 1055-1066, 2013.

[11] S. Ailawadhi, J. R. Mikhael, B. R. LaPlant et al., "Pomalidomide-dexamethasone in refractory multiple myeloma: long-term follow-up of a multi-cohort phase II clinical trial," Leukemia, vol. 32, no. 3, pp. 719-728, 2018.

[12] M. A. Dimopoulos, D. Dytfeld, S. Grosicki et al., "Elotuzumab plus pomalidomide and dexamethasone for multiple myeloma," New England Journal of Medicine, vol. 379, no. 19, pp. 1811-1822, 2018.

[13] J. F. San-Miguel, V. T. M. Hungria, S.-S. Yoon et al., "Overall survival of patients with relapsed multiple myeloma treated with panobinostat or placebo plus bortezomib and dexamethasone (the PANORAMA 1 trial): a randomised, placebo-controlled, phase 3 trial," The Lancet Haematology, vol. 3, no. 11, pp. e506-e515, 2016.

[14] J. F. San-Miguel, V. T. M. Hungria, S.-S. Yoon et al., "Panobinostat plus bortezomib and dexamethasone versus placebo plus bortezomib and dexamethasone in patients with relapsed or relapsed and refractory multiple myeloma: a multicentre, randomised, double-blind phase 3 trial," The Lancet Oncology, vol. 15, no. 11, pp. 1195-1206, 2014.

[15] D. Sivaraj, M. M. Green, and C. Gasparetto, "Panobinostat for the management of multiple myeloma," Future Oncology, vol. 13, no. 6, pp. 477-488, 2017.

[16] J. A. Zonder, A. F. Mohrbacher, S. Singhal et al., "A phase 1, multicenter, open-label, dose escalation study of elotuzumab in patients with advanced multiple myeloma," Blood, vol. 120, no. 3, pp. 552-559, 2012.

[17] P. G. Richardson, S. Jagannath, P. Moreau et al., "Elotuzumab in combination with lenalidomide and dexamethasone in patients with relapsed multiple myeloma: final phase 2 results from the randomised, open-label, phase $1 \mathrm{~b}-2$ doseescalation study," The Lancet Haematology, vol. 2, no. 12, pp. e516-e527, 2015.

[18] S. Lonial, M. Dimopoulos, A. Palumbo et al., "Elotuzumab therapy for relapsed or refractory multiple myeloma," New England Journal of Medicine, vol. 373, no. 7, pp. 621-631, 2015.

[19] S. Lonial, R. Vij, J.-L. Harousseau et al., "Elotuzumab in combination with lenalidomide and low-dose dexamethasone in relapsed or refractory multiple myeloma," Journal of Clinical Oncology, vol. 30, no. 16, pp. 1953-1959, 2012.

[20] A. Jakubowiak, M. Offidani, B. Pégourie et al., "Randomized phase 2 study: elotuzumab plus bortezomib/dexamethasone vs bortezomib/dexamethasone for relapsed/refractory MM," Blood, vol. 127, no. 23, pp. 2833-2840, 2016.

[21] A. J. Jakubowiak, D. M. Benson, W. Bensinger et al., "Phase I trial of anti-CS1 monoclonal antibody elotuzumab in combination with bortezomib in the treatment of relapsed/ refractory multiple myeloma," Journal of Clinical Oncology, vol. 30, no. 16, pp. 1960-1965, 2012.

[22] J. P. Laubach and P. G. Richardson, "CD38-targeted immunochemotherapy in refractory multiple myeloma: a new horizon," Clinical Cancer Research, vol. 21, no. 12, pp. 2660-2662, 2015.
[23] A. K. Nooka, J. L. Kaufman, C. C. Hofmeister et al., "Daratumumab in multiple myeloma," Cancer, vol. 125, no. 14, pp. 2364-2381, 2019.

[24] M. De Weers, Y.-T. Tai, M. S. van der Veer et al., "Daratumumab, a novel therapeutic human CD38 monoclonal antibody, induces killing of multiple myeloma and other hematological tumors," The Journal of Immunology, vol. 186, no. 3, pp. 1840-1848, 2011.

[25] M. B. Overdijk, S. Verploegen, M. Bögels et al., "Antibodymediated phagocytosis contributes to the anti-tumor activity of the therapeutic antibody daratumumab in lymphoma and multiple myeloma," $m A b s$, vol. 7, no. 2, pp. 311-320, 2015.

[26] M. B. Overdijk, J. H. M. Jansen, M. Nederend et al., "The therapeutic CD38 monoclonal antibody daratumumab induces programmed cell death via $\mathrm{fc} \gamma$ receptor-mediated cross-linking," The Journal of Immunology, vol. 197, no. 3, pp. 807-813, 2016.

[27] J. Krejcik, T. Casneuf, I. S. Nijhof et al., "Daratumumab depletes CD38+ immune regulatory cells, promotes T-cell expansion, and skews T-cell repertoire in multiple myeloma," Blood, vol. 128, no. 3, pp. 384-394, 2016.

[28] H. M. Lokhorst, T. Plesner, J. P. Laubach et al., "Targeting CD38 with daratumumab monotherapy in multiple myeloma," New England Journal of Medicine, vol. 373, no. 13, pp. 1207-1219, 2015.

[29] S. Lonial, B. M. Weiss, S. Z. Usmani et al., "Daratumumab monotherapy in patients with treatment-refractory multiple myeloma (SIRIUS): an open-label, randomised, phase 2 trial," The Lancet, vol. 387, no. 10027, pp. 1551-1560, 2016.

[30] S. Z. Usmani, B. M. Weiss, T. Plesner et al., "Clinical efficacy of daratumumab monotherapy in patients with heavily pretreated relapsed or refractory multiple myeloma," Blood, vol. 128 , no. 1, pp. 37-44, 2016.

[31] A. Palumbo, A. Chanan-Khan, K. Weisel et al., "Daratumumab, bortezomib, and dexamethasone for multiple myeloma," New England Journal of Medicine, vol. 375, no. 8, pp. 754-766, 2016.

[32] A. Spencer, S. Lentzsch, K. Weisel et al., "Daratumumab plus bortezomib and dexamethasone versus bortezomib and dexamethasone in relapsed or refractory multiple myeloma: updated analysis of CASTOR," Haematologica, vol. 103, no. 12, pp. 2079-2087, 2018.

[33] M. A. Dimopoulos, A. Oriol, H. Nahi et al., "Daratumumab, lenalidomide, and dexamethasone for multiple myeloma," New England Journal of Medicine, vol. 375, no. 14, pp. 1319-1331, 2016.

[34] M. A. Dimopoulos, J. San-Miguel, A. Belch et al., "Daratumumab plus lenalidomide and dexamethasone versus lenalidomide and dexamethasone in relapsed or refractory multiple myeloma: updated analysis of POLLUX," Haematologica, vol. 103, no. 12, pp. 2088-2096, 2018.

[35] A. Chari, A. Suvannasakha, J. W. Fay et al., "Daratumumab plus pomalidomide and dexamethasone in relapsed and/or refractory myeloma," Blood, vol. 130, no. 8, pp. 974-981, 2017.

[36] N. W. C. J. Van de Donk, P. Moreau, T. Plesner et al., "Clinical efficacy and management of monoclonal antibodies targeting CD38 and SLAMF7 in multiple myeloma," Blood, vol. 127, no. 6, pp. 681-695, 2016.

[37] H. M. Blommestein, C. H. Y. van Beurden-Tan, M. G. Franken, C. A. Uyl-de Groot, P. Sonneveld, and S. Zweegman, "Efficacy of first-line treatments for multiple myeloma patients not eligible for stem cell transplantation: a 
network meta-analysis," Haematologica, vol. 104, no. 5, pp. 1026-1035, 2019.

[38] A. Im and S. Z. Pavletic, "Immunotherapy in hematologic malignancies: past, present, and future," Journal of Hematology \& Oncology, vol. 10, no. 1, pp. 1-10, 2017.

[39] L. Falchi, A. Ferrajoli, I. Jacobs, and P. Nava-Parada, "An evidence-based review of anti-CD20 antibody-containing regimens for the treatment of patients with relapsed or refractory chronic lymphocytic leukemia, diffuse large B-cell lymphoma, or follicular lymphoma," Clinical Lymphoma Myeloma and Leukemia, vol. 18, no. 8, pp. 508-518, 2018.

[40] H. N. Abramson, "Monoclonal antibodies for the treatment of multiple myeloma: an update," International Journal of Molecular Sciences, vol. 19, no. 12, p. 3924, 2018.

[41] T. Ishida, "Therapeutic antibodies for multiple myeloma," Japanese Journal of Clinical Oncology, vol. 48, no. 11, pp. 957-963, 2018.

[42] P. G. Richardosn, S. Lonial, A. J. Jakubowiak, J. L. Harousseau, and K. C. Anderson, "Monoclonal antibodies in the treatment of multiple myeloma," British Journal of Haematology, vol. 154, no. 6, pp. 745-754, 2011.

[43] S. K. Kumar and K. C. Anderson, "Immune therapies in multiple myeloma," Clinical Cancer Research, vol. 22, no. 22, pp. 5453-5460, 2016.

[44] P. Kappor, P. T. Greipp, W. G. Morice, S. V. Rajkumar, T. E. Witzig, and P. R. Greipp, "Anti-CD20 monoclonal antibody therapy in multiple myeloma," British Journal of Haematology, vol. 141, no. 2, pp. 135-148, 2008.

[45] B. Huang, J. Li, J. Liu, and J. Gu, "CD20-positive multiple myeloma: can conventional chemotherapy still be used to achieve ideal outcome for these patients?," Leukemia \& Lymphoma, vol. 57, no. 2, pp. 335-340, 2016.

[46] G. Grigoriadis, M. Gilbertson, N. Came et al., "Is CD20 positive plasma cell myeloma a unique clinicopathological entity? A study of 40 cases and review of the literature," Pathology, vol. 44, no. 6, pp. 552-556, 2012.

[47] L. Moreno, C. Perez, A. Zabaleta, I. Manrique et al., "The mechanism of action of the anti-CD38 monoclonal antibody isatuximab in multiple myeloma," Clinical Cancer Research, vol. 25, no. 10, pp. 3176-3187, 2019.

[48] T. Martin, R. Baz, D. M. Benson et al., "A phase 1b study of isatuximab plus lenalidomide and dexamethasone for relapsed/refractory multiple myeloma," Blood, vol. 129, no. 25, pp. 3294-3303, 2017.

[49] J. Mikhael, P. Richardson, S. Z. Usmani et al., "A phase 1b study of isatuximab plus pomalidomide/dexamethasone in relapsed/refractory multiple myeloma," Blood, vol. 134, no. 2, pp. 123-133, 2019.

[50] R. Z. Orlowski, L. Gercheva, C. Williams et al., "A phase 2, randomized, double-blind, placebo-controlled study of siltuximab (anti-IL-6 mAb) and bortezomib versus bortezomib alone in patients with relapsed or refractory multiple myeloma," American Journal of Hematology, vol. 90, no. 1, pp. 42-49, 2015.

[51] P. M. Voorhees, R. F. Manges, P. Sonneveld et al., "A phase 2 multicentre study of siltuximab, an anti-interleukin-6 monoclonal antibody, in patients with relapsed or refractory multiple myeloma," British Journal of Haematology, vol. 161, no. 3, pp. 357-366, 2013.

[52] T. Jelinek, J. Mihalyova, M. Kascak, J. Duras, and R. Hajek, "PD-1/PD-L1 inhibitors in haematological malignancies: update 2017," Immunology, vol. 152, no. 3, pp. 357-371, 2017.

[53] J. Liu, A. Hamrouni, D. Wolowiec et al., "Plasma cells from multiple myeloma patients express B7-H1 (PD-L1) and increase expression after stimulation with IFN-\{gamma\} and TLR ligands via a MyD88-, TRAF6-, and MEK-dependent pathway," Blood, vol. 110, no. 1, pp. 296-304, 2007.

[54] A. Ray, D. S. Das, Y. Song et al., "Targeting PD1-PDL1 immune checkpoint in plasmacytoid dendritic cell interactions with $\mathrm{T}$ cells, natural killer cells and multiple myeloma cells," Leukemia, vol. 29, no. 6, pp. 1441-1444, 2015.

[55] H. Tamura, M. Ishibashi, T. Yamashita et al., "Marrow stromal cells induce B7-H1 expression on myeloma cells, generating aggressive characteristics in multiple myeloma," Leukemia, vol. 27, no. 2, pp. 464-472, 2013.

[56] G. Görgün, M. K. Samur, K. B. Cowens et al., "Lenalidomide Enhances immune checkpoint blockade-induced immune response in multiple myeloma," Clinical Cancer Research, vol. 21, no. 20, pp. 4607-4618, 2015.

[57] M. V. Dhodapkar, R. Sexton, R. Das et al., "Prospective analysis of antigen-specific immunity, stem-cell antigens, and immune checkpoints in monoclonal gammopathy," Blood, vol. 126, no. 22, pp. 2475-2478, 2015.

[58] B. Paiva, A. Azpilikueta, N. Puig et al., "PD-L1/PD-1 presence in the tumor microenvironment and activity of PD-1 blockade in multiple myeloma," Leukemia, vol. 29, no. 10, pp. 2110-2113, 2015.

[59] K. R. Kelly, C. M. Espitia, W. Zhao et al., "Oncolytic reovirus sensitizes multiple myeloma cells to anti-PD-L1 therapy," Leukemia, vol. 32, no. 1, pp. 230-233, 2018.

[60] D. M. Benson, C. E. Bakan, A. Mishra et al., "The PD-1/PDL1 axis modulates the natural killer cell versus multiple myeloma effect: a therapeutic target for CT-011, a novel monoclonal anti-PD-1 antibody," Blood, vol. 116, no. 13, pp. 2286-2294, 2010.

[61] S. Oliva, R. Troia, M. D’Agostino, M. Boccadoro, and F. Gay, "Promise and Pitfalls in the Use of PD-1/PD-L1 inhibitors in multiple myeloma," Frontiers in Immunology, vol. 9, no. 2749, pp. 1-8, 2018.

[62] T. Jelinek, B. Pavia, and R. Hajek, "Update on PD1/PD-L1 inhibitors in multiple myeloma," Frontiers in Immunology, vol. 9, no. 2431, pp. 1-13, 2018.

[63] A. M. Lesokhin, S. M. Ansell, P. Armand et al., "Nivolumab in patients with relapsed or refractory hematologic malignancy: preliminary results of a phase Ib study," Journal of Clinical Oncology, vol. 34, no. 23, pp. 2698-2704, 2016.

[64] S. Z. Usami, F. Schjesvold, A. Oriol et al., "Pembrolizumab plus lenalidomide and dexamethasone for patients with treatment-naïve multiple myeloma (KEYNOTE-185) randomized, open-label, phase 3 trial," The Lancet Haematology, vol. 6, no. 9, pp. e448-e458, 2019.

[65] M. V. Maetos, H. Blacklock, F. Schjesvold et al., "Pembrolizumab plus pomalidomide and dexamethasone for patients with relapsed or refractory multiple myeloma (KEYNOTE-183): a romdomised, open-label, phase 3 trial," The Lancet Haematology, vol. 6, no. 9, pp. e459-e469, 2019.

[66] P. A. Baeuerle and C. Reinhardt, "Bispecific T-cell engaging antibodies for cancer therapy," Cancer Research, vol. 69, no. 12, pp. 4941-4944, 2009.

[67] C. Caruso, "BiTE therapy active in multiple myeloma," Cancer Discovery, vol. 9, no. 2, pp. 157-158, 2019.

[68] S. Hipp, Y.-T. Tai, D. Blanset et al., "A novel BCMA/CD3 bispecific $\mathrm{T}$-cell engager for the treatment of multiple myeloma induces selective lysis in vitro and in vivo," Leukemia, vol. 31, no. 8, pp. 1743-1751, 2017.

[69] A. Goyos, C. M. li, P. Deegen et al., "Generation of half-life extended anti-BCMA Bite antibody construct compatible with once-weekly dosing anti-BCMA Bite antibody 
construct compatible with once-weekly dosing for the treatment of multiple myeloma (MM)," Blood, vol. 130, p. 5389, 2017.

[70] S. F. Cho, K. C. Anderson, and Y. T. Tai, "Targeting B cell maturation antigen (BCMA) in multiple myeloma: potential uses of BCMA-based immunotherapy," Frontiers in Immunology, vol. 9, no. 1821, pp. 1-15, 2018.

[71] J. R. Richter, C. O. Landgren, J. S. kauch et al., "Phase 1, multicenter open-label study of single-agent bispecific antibody t-cell engager GBR1342 in relapsed/refractory multiple myeloma," Journal of Clinical Oncology, vol. 36, no. 15 suppl, Article ID TPS3132, 2018.

[72] Y.-T. Tai, P. A. Mayes, C. Acharya et al., "Novel anti-B-cell maturation antigen antibody-drug conjugate (GSK2857916) selectively induces killing of multiple myeloma," Blood, vol. 123, no. 20, pp. 3128-3138, 2014.

[73] A. D. Cohen, R. Popat, S. Trude et al., "First in human study with GSK2857916, an antibody drug conjugated to microtubule-disrupting agent directed against B-cell maturation antigen (BCMA) in patients with relapsed/refractory multiple myeloma," Blood, vol. 128, no. 22, p. 1148, 2016.

[74] S. Trudel, N. Lendvai, R. Popat, P. M. Voorhees, B. Reeves, and E. N. Libby, "Deep and durable responses in patients (Pts) with relapsed/refractory multiple myeloma (MM) treated with monotherapy GSK2857916, an antibody drug conjugate against B-cell maturation antigen (BCMA): preliminary results from Part 2 of Study BMA117159," Blood, vol. 130, no. Suppl 1, p. 741, 2017.

[75] K. Hasegawa and N. Hosen, "Chimeric antigen receptor $\mathrm{T}$ cell therapy for multiple myeloma," Inflammation and Regeneration, vol. 39, no. 1, Article ID e16771, 2019.

[76] L. Mikkilineni and J. N. Kochenderfer, "Chimeric antigen receptor T-cell therapies for multiple myeloma," Blood, vol. 130, no. 24, pp. 2594-2602, 2018.

[77] P. Moreau, P. Sonneveld, M. Boccadoro et al., "CAR-T cell therapy for multiple myeloma: a consensus statement from the European myeloma network," Haematologica, 2019.

[78] R. O. Carpenter, M. O. Evbuomwan, S. Pittaluga et al., "B-cell maturation antigen is a promising target for adoptive $\mathrm{T}$-cell therapy of multiple myeloma," Clinical Cancer Research, vol. 19, no. 8, pp. 2048-2060, 2013.

[79] S. A. Ali, V. Shi, I. Maric et al., "T cells expressing an anti-Bcell maturation antigen chimeric antigen receptor cause remissions of multiple myeloma," Blood, vol. 128, no. 13, pp. 1688-1700, 2016.

[80] N. Raje, J. Berdeja, Y. Lin et al., "Anti-BCMA CAR T-cell therapy bb2121 in relapsed or refractory multiple myeloma," New England Journal of Medicine, vol. 380, no. 18, pp. 1726-1737, 2019.

[81] E. Drent, R. W. J. Groen, W. A. Noort et al., "Pre-clinical evaluation of CD38 chimeric antigen receptor engineered $\mathrm{T}$ cells for the treatment of multiple myeloma," Haematologica, vol. 101, no. 5, pp. 616-625, 2016.

[82] M. Casucci, B. Nicolis di Robilant, L. Falcone et al., "CD44v6-targeted T cells mediate potent antitumor effects against acute myeloid leukemia and multiple myeloma," Blood, vol. 122, no. 20, pp. 3461-3472, 2013.

[83] K. R. Kelly, A. Chanan-khan, G. Aomlo et al., "Indatuximab ravtansine (BT062) in combination with lenalidomide and low-dose dexamethasone in patients with relapsed and/or refractory multiple myeloma: clinical activity in Len/Dex refractory patients," Blood, vol. 122, p. 758, 2013.
[84] J. Berdeja, "Lorvotuzumab mertansine: antibody-drug-conjugate for $\mathrm{CD} 6^{+}$multiple myeloma," Frontiers in Bioscience, vol. 19, no. 1, pp. 163-170, 2014.

[85] M. Hussein, J. R. Berenson, R. Niesvizky et al., "A phase I multidose study of dacetuzumab (SGN-40; humanized antiCD40 monoclonal antibody) in patients with multiple myeloma," Haematologica, vol. 95, no. 5, pp. 845-848, 2010.

[86] W. Bensinger, R. T. Maziarz, S. Jagannath et al., "A phase 1 study of lucatumumab, a fully human anti-CD40 antagonist monoclonal antibody administered intravenously to patients with relapsed or refractory multiple myeloma," British Journal of Haematology, vol. 159, no. 1, pp. 58-66, 2012.

[87] J. L. Kaufman, R. Niesvizky, E. A. Stadtmauer et al., "Phase I, multicentre, dose-escalation trial of monotherapy with milatuzumab (humanized anti-CD74 monoclonal antibody) in relapsed or refractory multiple myeloma," British Journal of Haematology, vol. 163, no. 4, pp. 478-486, 2013.

[88] N. S. Raje, P. Moreau, E. Terpos et al., "Phase 2 study of tabalumab, a human anti-B-cell activating factor antibody, with bortezomib and dexamethasone in patients with previously treated multiple myeloma," British Journal of Haematology, vol. 176, no. 5, pp. 783-795, 2017.

[89] P. J. Hengeveld and M. J. Kersten, "B-cell activating factor in the pathophysiology of multiple myeloma: a target for therapy?," Blood Cancer Journal, vol. 5, no. 2, p. e282, 2015.

[90] L. Rasche, J. Duell, I. C. Castro et al., "GRP78-directed immunotherapy in relapsed or refractory multiple myeloma-results from a phase 1 trial with the monoclonal immunoglobulin M antibody PAT-SM6," Haematologica, vol. 100 , no. 3, pp. 377-384, 2015.

[91] P. Moreau, F. Cavallo, X. Leleu et al., "Phase I study of the anti-insulin-like growth factor 1 receptor (IGF-1R) monoclonal antibody, AVE1642, as single agent and in combination with bortezomib in patients with relapsed multiple myeloma," Leukemia, vol. 25, no. 5, pp. 872-874, 2011.

[92] S. Aichert, G. Juliusson, A. Johansson et al., "A single-arm, open-label, phase 2 clincal trial evaluating disease response following treatment with BI-505, a human anti-intracellular adhesion molecule-1 monoclonal antibody, in patients with smoldering multiple myeloma," Plos One, vol. 12, no. 2, Article ID e0171205, 2017.

[93] B. Fleischer, "CD26: a surface protease involved in T-cell activation,” Immunology Today, vol. 15, no. 4, pp. 180-184, 1994.

[94] T. Tanaka, D. Camerini, B. Seed et al., "Cloning and functional expression of the T cell activation antigen CD26," The Journal of Immunology, vol. 149, no. 2, pp. 881-886, 1992.

[95] R. Dong, K. Tachibana, M. Hegen et al., "Correlation of the epitopes defined by anti-CD26 mAbs and CD26 function," Molecular Immunology, vol. 35, no. 1, pp. 13-21, 1998.

[96] Y. Torimoto, N. H. Dang, T. Tanaka, C. Prado, S. F. Schlossman, and C. Morimoto, "Biochemical characterization of CD26 (dipeptidyl peptidase IV): functional comparison of distinct epitopes recognized by various antiCD26 monoclonal antibodies," Molecular Immunology, vol. 29, no. 2, pp. 183-192, 1992.

[97] K. Ohnuma, N. Takahashi, T. Tamochu, O. Hosono, N. H. Dang, and C. Morimoto, "Role of CD26/dipeptidyl peptidase IV in human T cell activation and function," Frontiers in Bioscience, vol. 13, no. 13, pp. 2299-2310, 2008.

[98] P. A. Havre, M. Abe, Y. Urasaki, K. Ohnuma, C. Morimoto, and N. H. Dang, "The role of CD26/dipeptidyl peptidase IV in cancer," Frontiers in Bioscience, vol. 13, no. 13, pp. 1634-1645, 2008. 
[99] H. Nishida, M. Hayashi, C. Morimoto, M. Sakamoto, and T. Yamada, "CD26 is a potential therapeutic target by humanized anti-body for the treatment of multiple myeloma," Blood Cancer Journal, vol. 8, no. 11, pp. 1-17, 2018.

[100] H. Nishida, H. Suzuki, H. Madokoro et al., "Blockade of CD26 signaling inhibits human osteoclast development," Journal of Bone and Mineral Research, vol. 29, no. 11, pp. 2439-2455, 2014. 


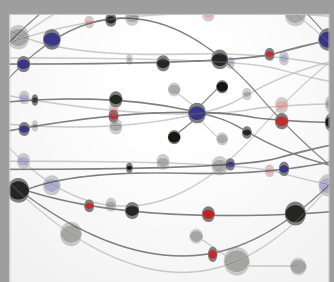

The Scientific World Journal
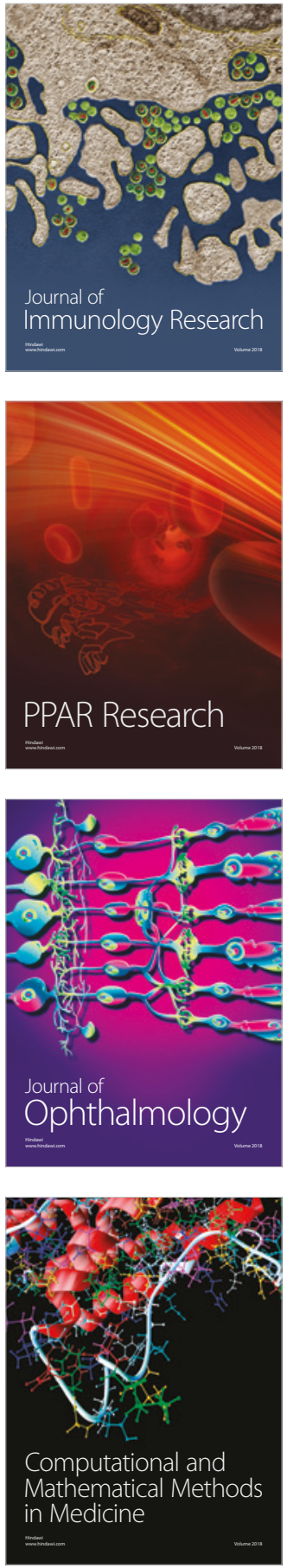

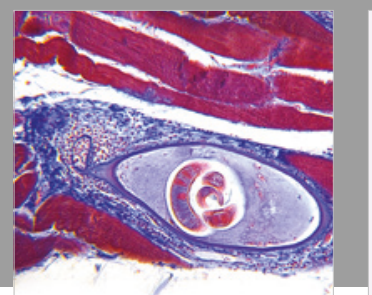

Gastroenterology Research and Practice

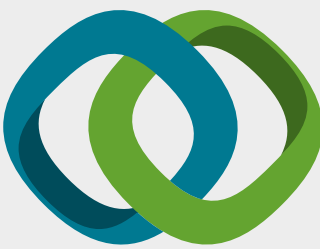

\section{Hindawi}

Submit your manuscripts at

www.hindawi.com
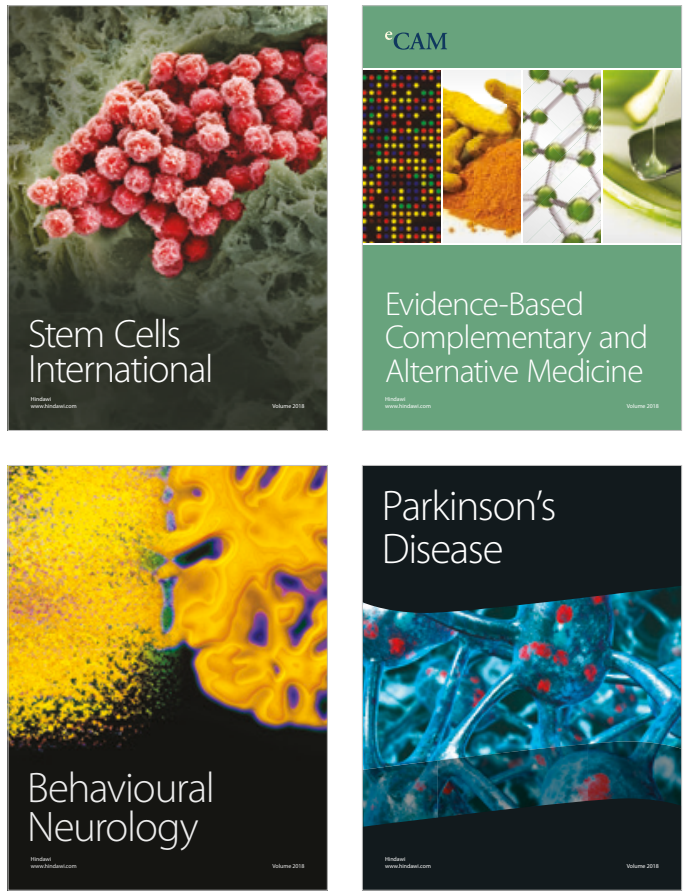

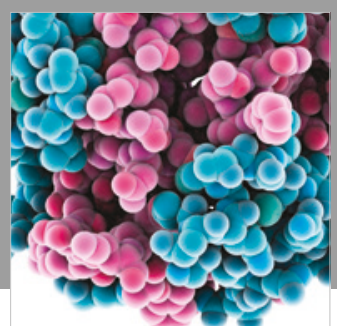

ournal of

Diabetes Research

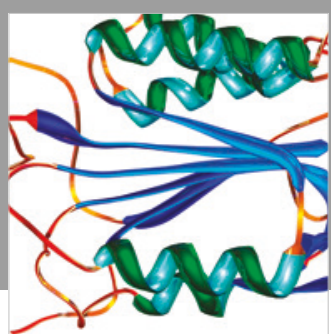

Disease Markers
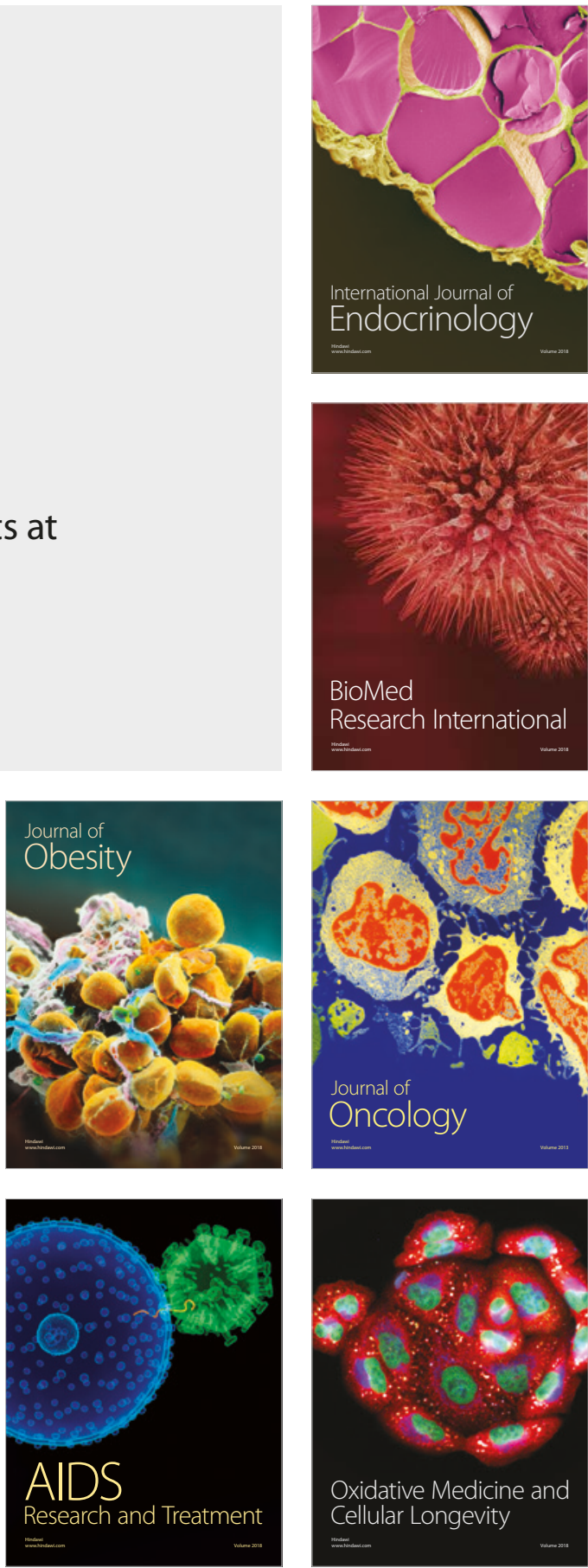